\title{
Pediatric anti-HMGCR necrotizing myopathy: diagnostic challenges and literature review
}

\author{
Daniele Velardo ${ }^{1}$ (1) \& Irene Faravelli ${ }^{2}$ \& Claudia Cinnante ${ }^{3}$ \& Maurizio Moggio ${ }^{1}$ \& Giacomo Pietro Comi ${ }^{1,2}$
}

\section{Dear Editor-in-Chief,}

A 7-year-old boy presented with a 2-month subtle onset, progressive lower limb weakness with marked difficulty in getting up from the ground. Creatine kinase (CK) level at presentation was 10,000 U/L. No previous infection was reported, and family history was apparently negative for neuromuscular disorders. Neurological exam revealed proximal weakness of all limbs (Medical Research Council [MRC] grade 4, more pronounced at the level of iliopsoas muscles, MRC grade 3) and need for one-hand support to get up from the ground. Multiplex Ligation-Dependent Probe Amplification (MLPA) analysis of Duchenne Muscular Dystrophy (DMD) gene showed no deletion or duplication. Therefore, the patient underwent muscle biopsy (left biceps brachii), which showed a dystrophic pattern with numerous necrotizing and rare regenerating fibers (Fig. 1). In immunohistochemistry analyses, dystrophin and beta-sarcoglycan binding alterations were observed in rare fibers, but western blot analyses highlighted dystrophin, alpha-dystroglycan, calpain-3, dysferlin, and $\alpha$ - to $\delta$-sarcoglycans normal molecular weights and amounts. Fukutin-related protein and acid alphaglucosidase genetic investigation also resulted normal. Limbgirdle muscular dystrophy (LGMD) next generation sequencing (NGS) panel did not reveal any known pathogenic mutations. Lower limb muscle magnetic resonance imaging (MRI)

* Daniele Velardo

velardo.daniele@gmail.com

1 Neuromuscular and Rare Diseases Unit, Department of Neuroscience, Foundation IRCCS Ca' Granda Ospedale Maggiore Policlinico, Via Francesco Sforza 35, 20122 Milan, Italy

2 Neurology Unit, Neuroscience Section, Department of Pathophysiology and Transplantation, Dino Ferrari Centre, Foundation IRCCS Ca' Granda Ospedale Maggiore Policlinico, University of Milan, Via Francesco Sforza 35, 20122 Milan, Italy

3 Neuroradiology Unit, Foundation IRCCS Ca' Granda Ospedale Maggiore Policlinico, Via Francesco Sforza 35, 20122 Milan, Italy showed relative hypotrophy of proximal muscles (i.e., gluteus maximus, thigh adductors, and posterior compartment of thigh) with only mild fibro-adipose substitution but without significant signs of edema (Fig. 2). Echocardiography was normal.

During the next 4 months, while work-up results were pending, no therapy was administered. However, the patient complained of rapidly progressive fatigue, weight reduction with mild swallowing dysfunction, difficulty in climbing stairs, and loss of running ability. Simultaneously, the patient developed dermatologic manifestations characterized by small bald patches of alopecia on the scalp, self-limiting recurring roughening, and cracking of hand fingertips and erythematous papular lesions on ears and extensor surface of elbows and knees (Fig. 1).

Four months after the first assessment, neurological examination evidenced four limb muscles weakness (MRC grade between 3 and 4) and diffuse loss of muscular tone involving axial muscles of trunk and head, along with complete Gowers' sign. Spirometry showed restrictive ventilatory defect of moderate severity (FVC 65\%, FEV1 76\%, FEV1/FVC 100\% of predicted). The patient scored 18/34 at the North Star Ambulatory Assessment (NSAA) and reached $375 \mathrm{~m}$ at the 6 Minutes Walking Test (6MWT). CK level was only slightly reduced (8501 U/L, reference values 38-247). Re-evaluation of muscle biopsy highlighted sarcolemmal and sarcoplasmic positivity of anti-major histocompatibility complex (MHC)-I antibodies in some fibers and complement deposition on muscle fiber membranes and capillaries (Fig. 1). Autoimmune serologic screening revealed anti-3-hydroxy-3-methylglutaryl-coenzyme A reductase (HMGCR) antibody positivity (268 arbitrary units [AU], reference level $<20$ ), while all the other myositis-specific and myositis-associated autoantibodies tested were negative (i.e., anti-Jo1, anti-PL-7, antiPL-12, anti-Mi-2, anti-SRP-S4, anti-Scl70, anti-Ro/SSA, anti-La/SSB, anti-PM/Scl, and anti-Ku). Four limbs MRI showed the appearance of short-tau inversion-recovery (STIR) signal hyperintensity, particularly at the level of distal leg muscles (i.e., triceps surae, tibial posterior, and peroneus 


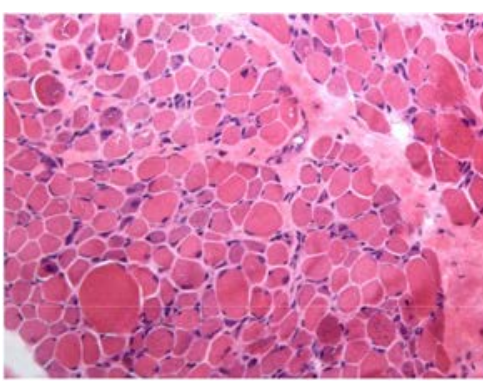

a

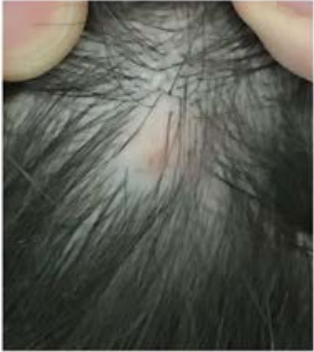

d

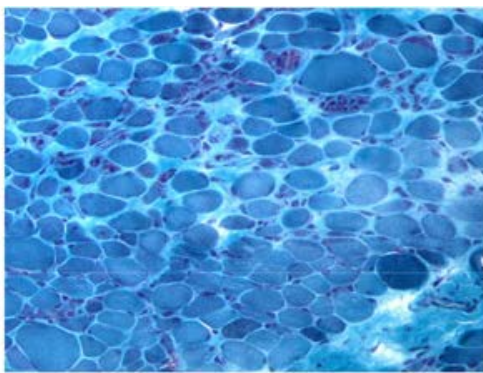

b

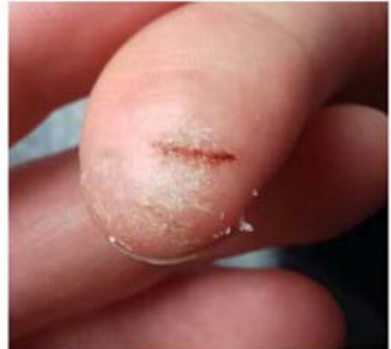

e

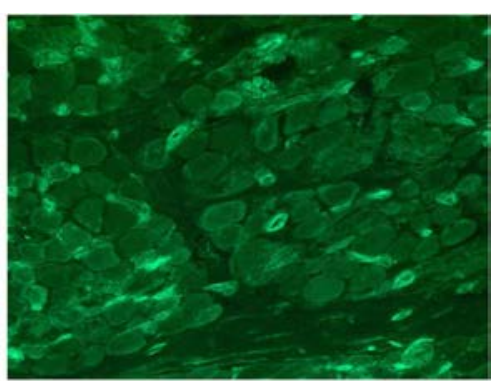

C
Fig. 1 Muscle biopsy: hematoxylin and eosin staining (a) showing increase in endomysial and perimysial connective tissue, varied muscle fiber diameter with numerous small rounded and one hypertrophic fiber, necrotic fibers, and occasional internal nuclei; Gomori trichrome (b) confirming increase in endomysial connective tissue and necrotic fibers;

longus muscles) (Fig. 3). Transthoracic echocardiography showed hypokinesia and rigidity of the interventricular septum basal segments, with normal left ventricle (LV) dimension and function parameters (LV end-diastolic diameter $34 \mathrm{~mm}, \mathrm{LV}$ endocardial fractional shortening 36\%, LV ejection fraction 67\%).

The patient was treated with intravenous steroids (methylprednisolone $15 \mathrm{mg} / \mathrm{kg}$ for 5 days) followed by slow tapering oral prednisone (starting with $1 \mathrm{mg} / \mathrm{kg}$ once daily), with initial progressive improvement of the symptoms and normalization of cardiac imaging.
MHC-1 immunofluorescence (c) showing sarcolemmal and cytoplasmic positivity in some fibers; cutaneous manifestations: small bald patch of alopecia on the scalp (d); roughening and cracking of thumb fingertip (e); and erythematous papular lesions on extensor surface of right knee (f)

One month later, the therapy was implemented with administration of intravenous immunoglobulins (IVIg, $2 \mathrm{~g} / \mathrm{kg}$ in divided doses over 4 consecutive days) every 2 months for three times, with further functional recovery. Two months after the last immunoglobulin infusion, a clinical, radiological, and biochemical re-evaluation was carried out: the patient scored 27/34 at the NSAA and was able to cover $488 \mathrm{~m}$ at the 6MWT. Cutaneous manifestations had disappeared. Nevertheless, the patient still exhibited mild (MRC grade 4) upper limbs and moderate lower limbs (MRC grade 3-4) proximal weakness. Four limbs MRI excluded progression
Fig. 2 Clinical onset MRI: MRI TSE T1 axial images on pelvic girdle (a), thigh (b), and shoulder girdle and arm (c), showing a diffuse muscular hypotrophy, more pronounced at the level of gluteus maximus (a, black arrow), at the mesial and posterior compartment of the thigh (b, white circle), and at the triceps (c, white arrow). No significant fatty degeneration was seen. No edema detectable on axial STIR-T2 images on thigh muscle (d)

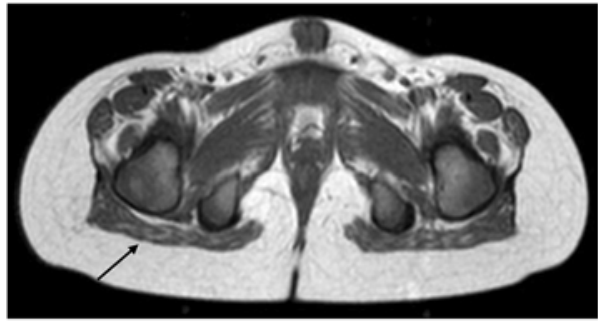

a

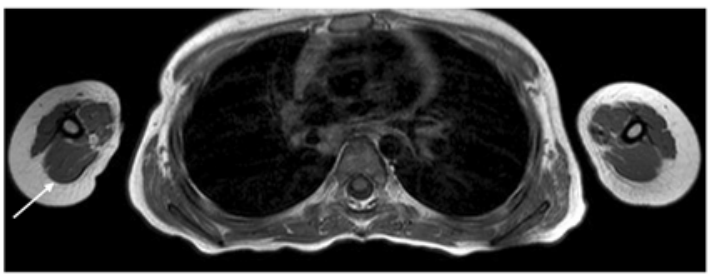

C

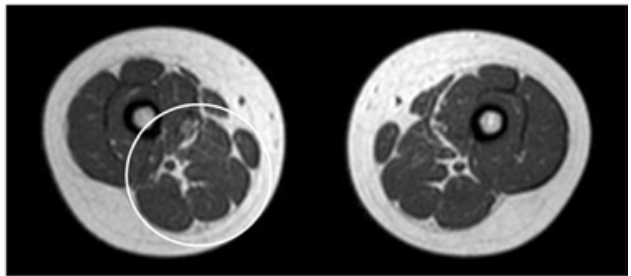

b

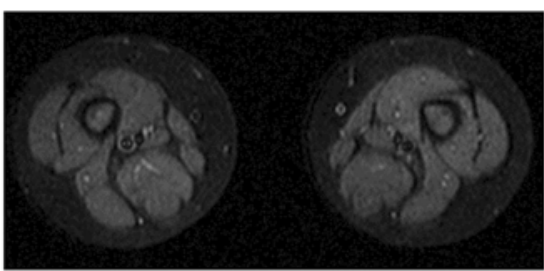

d 


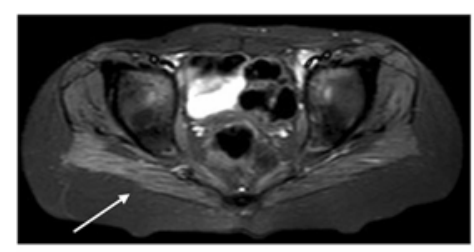

a

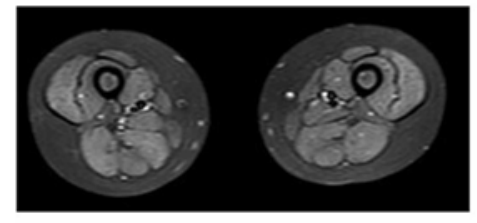

b

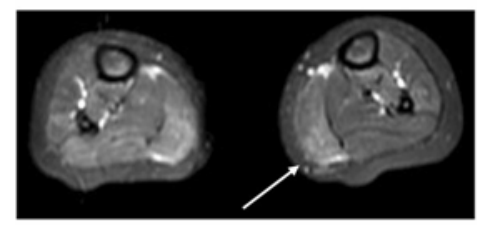

C

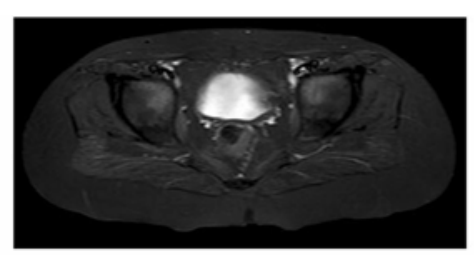

d

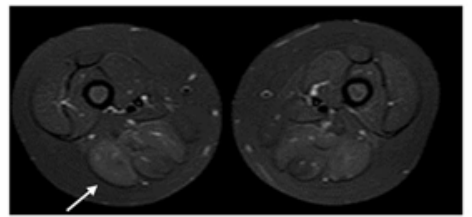

e

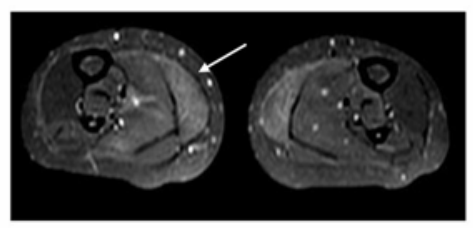

f

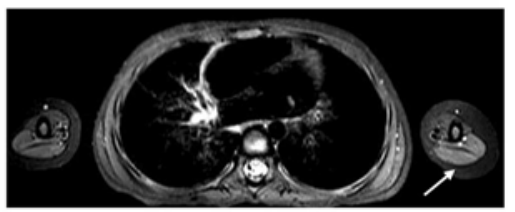

g

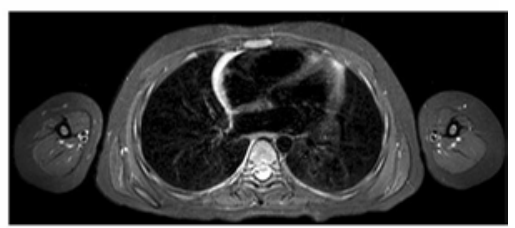

h
Fig. 3 Pre- and post-treatment MRI. MRI STIR T2 axial images on pelvic girdle, thigh and calf, shoulder girdle, and arm before the treatment (a-b-c-g) and after the treatment (d-e-f-h). MRI images before the treatment showing a hyperintensity signal, suggestive for edema and inflammation, at the level of right gluteus maximus (a, white arrow), lighter at the thigh muscles (b), and more emphatic at the calf muscles, especially at the medial head of the gastrocnemius, even bulgy (c, white arrow).At the level of the upper arm, a slight hyperintensity signal was seen at the triceps (g, white arrow). After the treatment, a general

of fibro-adipose substitution and muscle hypotrophy, while showing regression of upper limbs STIR sequences signal hyperintensity findings. However, MRI confirmed mild muscle edema of posterior compartment of thighs and legs. Serum anti-HMGCR antibodies level was substantially unchanged (251.9 AU, reference level < 20), whereas CK level was rising again (5966 U/L) compared with that measured immediately before IVIg third cycle (i.e., 3624 U/L).

In view of these data, the patient was treated with intravenous steroid (methylprednisolone $30 \mathrm{mg} / \mathrm{kg}$ for 3 days) followed by tapering oral prednisone ( $2 \mathrm{mg} / \mathrm{kg} /$ day as 2 divided doses) and subcutaneous methotrexate ( $0.5 \mathrm{mg} / \mathrm{kg} /$ week). Follow-up is currently ongoing.

Anti-HMGCR autoantibody-positive immune-mediated necrotizing myopathy (IMNM) was first recognized in adult patients with a history of statin exposure. The clinicopathologic spectrum of the disease is constantly updated, including statin-unexposed patients, childhood-onset cases, and pediatric and adult patients with a LGMD phenotype[1]. In October 2016, a new classification of IMNM was proposed, including expert advice regarding treatment recommendations for pediatric cases[2]. The consensus was reached through expert opinions and available scientific reports [3, 4]. The slow progression of the disease already suggested that anti-HMGCR IMNM should be considered in the differential diagnosis in improvement of the radiological pattern was seen, though not completely solved: a slight hyperintensity signal was seen at the level of the gluteus maximus (d) and the posterior compartment of the thigh muscles (e, white arrow) and at the level of the mesial compartment of the calf (f, white arrow). At the level of the arm, the edema was completely solved (h). The selectivity pattern is slightly asymmetrical and with a disto-proximal gradient. No significant progression of fatty degeneration was seen on post-treatment MRI TSE T1 images (not shown)

children with idiopathic limb-girdle myopathy and silent neuromuscular family history.

In 2017, at least four reports described further cases of pe diatr ic anti-HMGCR-asso cia ted necrotizing myopathies[5-8]. All these studies described the more common slow disease progression compared with adult patients, the presence of skin involvement, the relatively less consistent cardiac and respiratory impairment, the frequent diagnostic delay, and the poor response to therapy.

Pathology plays a pivotal role in the diagnosis: myofiber degeneration and regeneration represent a practically constant feature in pediatric cases, and most biopsies include different degrees of endomysial fibrosis, whereas prominent cellular infiltration is not a typical feature. MHC-1 immunostaining can be increased in non-degenerating fibers, although with a weaker and patchy signal, which in some cases may even be absent, as compared with other inflammatory myopathies [5, 9].

MRI is by now an extensively used instrument for the diagnosis and follow-up of myositis. In the literature, MRI showed STIR signal hyperintensity of leg muscles in all pediatric patients tested before treatment; only in one case muscle MRI was performed after therapy, showing significant reduction in STIR signal [5, 6]. Several immunosuppressive therapies have been attempted, but clinical remission has been 
achieved only in two patients [7]. Most of cases required a chronic multidrug treatment resulting in partial remission, confirming the poorer response to treatment compared with the adult population, at least in a short-term treatment followup.

In our case, the initial diagnosis was challenging. Considering the age of onset, the CK level, and the clinical presentation, we initially decided not to submit the child to an invasive diagnostic procedure and performed MLPA analysis of DMD gene. Further investigations became necessary due to the negativity of the genetic test, but the initial absence of associated systemic features, the muscle histological picture displaying a predominant dystrophic pattern with associated necrotizing fibers, and the MRI findings showing complete lack of muscle edema were further confounding elements. Of note, the MRI performed just 2 months after the onset of symptoms did not show any obvious alterations in STIR sequences, even in the clinically involved muscles; this could be due to the early timing of the investigation. Moreover, MRI scans carried out immediately before the start of treatment exhibited the appearance of increased signal intensity on STIR images in clinically less involved muscles (i.e., posterior compartment of legs). These observations demonstrate a delayed positivization of MRI and no precise match between clinical involvement and MRI STIR hyperintensity degree.

It is also interesting to underline the rapid clinical evolution of our case, with early involvement of the bulbar, cardiac, and respiratory muscles; in particular, echocardiographic alterations have not previously been reported in pediatric patients with anti-HMGCR IMNM, expanding the phenotypic spectrum of the disease.

We decided to treat our patient with pulse steroids followed by oral tapering and the addition of intravenous immunoglobulin within 1 month, in compliance with the European NeuroMuscular Centre recommendations [2]. However, this first-line treatment resulted in an incomplete response, as confirmed by the new rise in muscle enzymes and the persistence of clinical signs. MRI images confirmed a partial regression of signs possibly related to muscle edema, thus suggesting that radiological follow-up may be instrumental in the follow-up of these patients. As previously described in adult statinunexposed patients [10,11], autoantibody levels do not decline with immunotherapy, and there is no correlation of antibody levels with CK or clinical and radiological assessments.

Our case report confirms that pediatric anti-HMGCR necrotizing myopathy is a systemic disease, also involving cardiac muscle. Immunohistochemical muscle biopsy staining for MHC-1 and HMGCR autoantibody testing should be part of the initial evaluation in all pediatric patients with clinically and pathologically suspected muscular dystrophy and unrevealing genetic testing or negative family history. An initially negative muscle MRI, especially if performed shortly after the onset of symptoms, does not exclude the diagnosis; the exam should be repeated later in the clinical course and possibly used in the long-term monitoring of these patients. Cardiological assessments should be part of the diagnostic and follow-up workout of these patients. Early diagnosis and treatment could be a valuable approach in this disease, but there is still a substantial need for new data about long-term efficacy of currently available drugs and new therapeutic approaches.

Author contributions DV, IF, CC, MM, and GC participated in the acquisition and analysis of data, in the conception of the manuscript, in drafting manuscript or figures, and in the critical revision.

\section{Compliance with ethical standards}

Conflict of interest The authors declare that they have no conflict of interest.

Ethical standards This case report is in accordance with the ethical standards laid down in the declaration of Helsinki and its later amendments.

\section{References}

1. Mohassel P, Mammen AL (2018) Anti-HMGCR Myopathy. J Neuromuscul Dis 5(1):11-20 Review

2. Allenbach Y, Mammen AL, Benveniste O, Stenzel W (2018) Immune-Mediated Necrotizing Myopathies Working Group. 224th ENMC International Workshop: Clinico-sero-pathological classification of immune-mediated necrotizing myopathies Zandvoort, The Netherlands, 14-16 October 2016. Neuromuscul Disord 28(1):87-99

3. Allenbach Y, Drouot L, Rigolet A, Charuel JL, Jouen F, Romero NB, Maisonobe T, Dubourg O, Behin A, Laforet P, Stojkovic T, Eymard B, Costedoat-Chalumeau N, Campana-Salort E, Tournadre A, Musset L, Bader-Meunier B, Kone-Paut I, Sibilia J, Servais L, Fain O, Larroche C, Diot E, Terrier B, De Paz R, Dossier A, Menard D, Morati C, Roux M, Ferrer X, Martinet J, Besnard S, Bellance R, Cacoub P, Arnaud L, Grosbois B, Herson S, Boyer O, Benveniste O, French Myositis Network (2014) Anti-HMGCR autoantibodies in European patients with autoimmune necrotizing myopathies: inconstant exposure to statin. Medicine (Baltimore) 93(3):150-157

4. Mammen AL, Chung T, Christopher-Stine L, Rosen P, Rosen A, Doering KR, Casciola-Rosen LA (2011) Autoantibodies against 3hydroxy-3-methylglutaryl-coenzyme A reductase in patients with statin-associated autoimmune myopathy. Arthritis Rheum 63(3): 713-721

5. Liang WC, Uruha A, Suzuki S, Murakami N, Takeshita E, Chen WZ, Jong YJ, Endo Y, Komaki H, Fujii T, Kawano Y, MoriYoshimura M, Oya Y, Xi J, Zhu W, Zhao C, Watanabe Y, Ikemoto K, Nishikawa A, Hamanaka K, Mitsuhashi S, Suzuki N, Nishino I (2017) Pediatric necrotizing myopathy associated with anti-3-hydroxy-3-methylglutaryl-coenzyme A reductase antibodies. Rheumatology (Oxford) 56(2):287-293

6. Mohassel P, Foley AR, Donkervoort S, Fequiere PR, Pak K, Bönnemann CG, Mammen AL (2017) Anti-3-hydroxy-3-methylglutaryl-coenzyme a reductase necrotizing myopathy masquerading as a muscular dystrophy in a child. Muscle Nerve 56(6):11771181

7. Tansley SL, Betteridge ZE, Simou S, Jacques TS, Pilkington C, Wood M, Warrier K, Wedderburn LR, NJ MH (2017) Juvenile 
Dermatomyositis Research Group. Anti-HMGCR autoantibodies in juvenile idiopathic inflammatory myopathies identify a rare but clinically important subset of patients. J Rheumatol 44(4):488-492

8. Kishi T, Rider LG, Pak K, Barillas-Arias L, Henrickson M, McCarthy PL, Shaham B, Weiss PF, Horkayne-Szakaly I, Targoff IN, Miller FW, Mammen AL, Childhood Myositis Heterogeneity Study Group (2017) Association of anti-3-hydroxy-3-methylglutaryl-coenzyme A reductase autoantibodies with DRB1*07:01 and severe myositis in juvenile myositis patients. Arthritis Care Res 69(7):1088-1094

9. Mohassel P, Landon-Cardinal O, Foley AR, Donkervoort S, Pak KS, Wahl C, Shebert RT, Harper A, Fequiere P, Meriggioli M, Toro C, Drachman D, Allenbach Y, Benveniste O, Béhin A, Eymard B, Lafôret P, Stojkovic T, Mammen AL, Bönnemann CG (2018) Anti-HMGCR myopathy may resemble limb-girdle muscular dystrophy. Neurol Neuroimmunol Neuroinflamm 6(1): e523

10. Werner JL, Christopher-Stine L, Ghazarian SR, Pak KS, Kus JE, Daya NR et al (2012) Antibody levels correlate with creatine kinase levels and strength in anti-3-hydroxy-3-methylglutaryl-coenzyme A reductase-associated autoimmune myopathy. Arthritis Rheum 64:4087-4093

11. Ge Y, Lu X, Peng Q, Shu X, Wang G (2015) Clinical characteristics of anti-3-hydroxy-3-methylglutaryl coenzyme A reductase antibodies in Chinese patients with idiopathic inflammatory myopathies. PLoS One 10(10):e0141616 